\title{
Scaling laws for random walks in long-range correlated disordered media
}

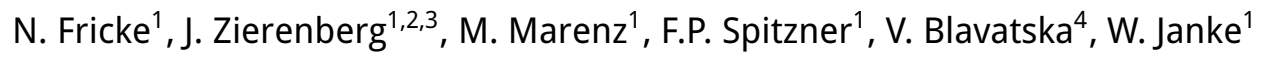 \\ ${ }^{1}$ Institut für Theoretische Physik, Universität Leipzig, Postfach 100 920, D-04009 Leipzig, Germany \\ 2 Bernstein Center for Computational Neuroscience, Am Fassberg 17, D-37077 Göttingen, Germany \\ 3 Max Planck Institute for Dynamics and Self-Organsization, Am Fassberg 17, D-37077 Göttingen, Germany \\ ${ }^{4}$ Institute for Condensed Matter Physics of the National Academy of Sciences of Ukraine, \\ 1 Svientsitskii St., 79011 Lviv, Ukraine
}

Received January 27, 2017, in final form March 14, 2017

\begin{abstract}
We study the scaling laws of diffusion in two-dimensional media with long-range correlated disorder through exact enumeration of random walks. The disordered medium is modelled by percolation clusters with correlations decaying with the distance as a power law, $r^{-a}$, generated with the improved Fourier filtering method. To characterize this type of disorder, we determine the percolation threshold $p_{\mathrm{c}}$ by investigating cluster-wrapping probabilities. At $p_{\mathrm{c}}$, we estimate the (sub-diffusive) walk dimension $d_{\mathrm{w}}$ for different correlation exponents $a$. Above $p_{c}$, our results suggest a normal random walk behavior for weak correlations, whereas anomalous diffusion cannot be ruled out in the strongly correlated case, i.e., for small $a$.
\end{abstract}

Key words: long-range correlated disorder, critical percolation clusters, random walks, exact enumerations, scaling laws

PACS: 05.70.Jk, 64.60.al, 64.60.De

\section{Introduction}

Structural disorder has a strong effect on a wide range of physical processes and can alter the behavior of systems such as magnets or polymers [1]2]. Examples for disordered systems are porous materials, which often have fractal structure. This may lead to anomalous diffusive transport [3] 4], an aspect that is relevant to problems ranging from oil recovery through porous rocks [5] 6] and the dynamics of fluids in disordered media [7 8] to transport processes in crowded biological cells [9, 10].

Disordered systems are conveniently described in the framework of lattice models with randomly positioned defects. Already a small amount of defects can drive the critical behavior of magnetic systems into a new "disordered" universality class [11], but the scaling properties of diffusion (usually modelled by random walks) and polymers (usually modelled by self-avoiding random walks) are thought to be unaffected in this case [12, 13]. For a larger amount of defects, near the percolation threshold of the nondefect sites, clusters of connected sites become fractal, i.e., self-similar objects devoid of a characteristic length scale. The case where the defects are uncorrelated is a classic textbook model, whose properties have been studied extensively [14]. Right at the percolation point, even diffusion and polymer statistics show a modified, anomalous behavior, which has been extensively studied using field-theoretic renormalization group methods [15, 16] and computer simulations [17]. For recent examples see [18-25] and further references therein.

In nature, however, inhomogeneities are frequently not distributed totally at random but tend to be correlated over large distances. To understand the impact of this, it is useful to consider the limiting case where correlations decay asymptotically as a power law (rather than exponentially) with distance $r$ :

$$
C(r) \sim r^{-a} .
$$


If the correlation exponent $a$ is smaller than the spatial dimension $d$, the correlations are considered as long-range or "infinite". This problem has first been investigated in the context of spin systems and later on for percolation [26, 27]. The relevance of the disorder was shown to be characterized by an extension of the Harris criterion: the critical behavior of the system changes if the minimum of $d$ and $a$ is smaller than $2 / v$, where $v$ denotes the correlation-length exponent for a pure system. Furthermore, it was argued that in the regime of long-range correlations, the critical correlation-length exponent for strong disorder is always given by $2 / a$. This result is still slightly controversial [28-30], but it has, to some extent, been supported by numerical investigations [31,32]. These studies made use of the Fourier filtering method (FFM) [31, 33, 35] to generate power-law correlated disorder and have yielded estimates for the values of various critical exponents and fractal dimensions characterizing the disordered media.

Systems confined to media with long-range correlated disorder show a very interesting behavior, but still comparatively few properties are fully understood to date. The investigated examples include magnetic materials [36,41] and macromolecular systems [42,44], again mainly using field-theoretic renormalization group methods [15, 16] and computer simulations [17]. Long-range correlations have also been shown to have an impact on the conductance of critical clusters and on their properties as a medium for diffusion. However, these investigations have so far focused on the system at criticality, while the situation with fewer defects has largely been disregarded. This regime is of little interest for the uncorrelated case, where the behavior should be the same as in the undiluted system. However, large fluctuations caused by the long-range correlations may have a non-trivial impact on the system's dynamic exponents and give rise to anomalous diffusion. In the context of continuum percolation, fluid dynamics and diffusive transport in correlated disordered media have recently been investigated by simulations and experiment [7] 8], and correlated random potentials could be realized experimentally with laser speckle patterns 45$]$.

The remainder of the article is organized as follows: in section 2.1 we closely follow the procedure of [35] and use the (improved) FFM in order to first generate continuous variables with varying degree of correlation $a$, which are then mapped to correlated discrete values as illustrated in figure 1 In section 2.2. we determine the site-percolation threshold $p_{\mathrm{c}}$ for a square lattice as a function of $a$. In section 3 we use exact enumerations of random walks in the thus generated long-range correlated disordered medium to study diffusion and analyze the scaling behavior for the quenched disorder average of the mean square displacement. This is done for varying degrees of correlation controlled by the exponent $a$ and different concentrations $p$. The conclusions of our work and prospects for future investigations are contained in section 4

\section{Long-range correlated percolation clusters}

\subsection{Generation of power-law correlations}

We consider a two-dimensional square lattice with $L \times L$ sites labeled by $\vec{x}$. The goal is to obtain discrete binary site values, $s_{\vec{x}} \in\{0,1\}$, that exhibit a power-law correlation. This can be achieved in a twostep process. First, one uses the modified Fourier filtering method (FFM) [34, 35] to generate continuous Gaussian site variables $\varphi_{\vec{x}}$ that are power-law correlated, i.e.,

$$
\left\langle\varphi_{\vec{x}} \varphi_{\vec{x}+\vec{r}}\right\rangle \sim r^{-a},
$$

for sufficiently large distances $r$. In the second step, the continuous variables $\varphi_{\vec{x}}$ are mapped to discrete values $\{0,1\}$.

The method starts with uncorrelated random numbers $u_{\vec{x}}$ drawn from a Gaussian distribution with variance one and mean zero, which are distributed onto the two-dimensional lattice. The key idea now is to multiply the Fourier coefficients $u_{\vec{q}}$ with the square root of the spectral density $S_{\vec{q}}$, which is the Fourier transform of the desired correlation function:

$$
\varphi_{\vec{q}}=\sqrt{S_{\vec{q}}} u_{\vec{q}}
$$

The inverse Fourier transform should then yield coefficients $\varphi_{\vec{x}}$ with the desired power-law correlations. The density distribution of the values, $P\left(\varphi_{\vec{x}}\right)$, is again Gaussian in the asymptotic limit of large systems 

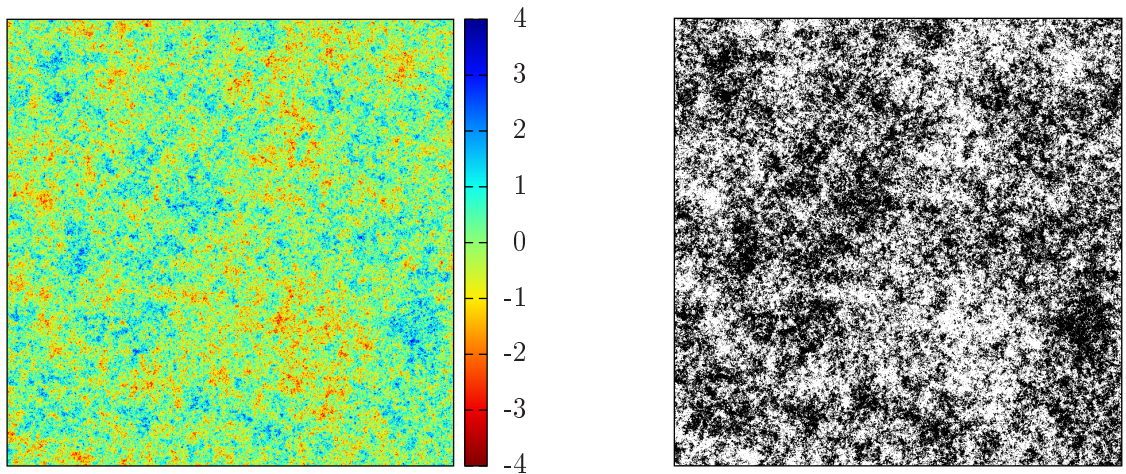

Figure 1. (Color online) Correlated continuous variables on a $2^{11} \times 2^{11}$ lattice for a correlation exponent $a=0.5$ (left-hand) and corresponding discrete binary variables at the percolation threshold ( $p=0.5209$ ) with defects shown in black (right-hand).

and/or many disorder realizations [34]. The coefficients $u_{\vec{q}}$ and $\varphi_{\vec{x}}$ can be efficiently calculated using the discrete fast Fourier transform (FFT) algorithm [46].

The irrelevant singularity of the correlation function 2.1 at $r=0$ poses technical problems. These can be overcome by introducing a function with the same asymptotic large-distance limit:

$$
C(r)=\left(1+r^{2}\right)^{-a / 2} \stackrel{r \gg 1}{\longrightarrow} r^{-a} .
$$

Using a continuum approximation, the spectral density can then be calculated analytically [35]. In $d=2$ dimensions, the result is

$$
S_{\vec{q}}=\frac{2 \pi}{\Gamma\left(\frac{a}{2}\right)}\left(\frac{|\vec{q}|}{2}\right)^{\frac{a}{2}-1} K_{\frac{a}{2}-1}(|\vec{q}|),
$$

where $K_{\beta}(q)$ is the modified Bessel function of the order $\beta$. This is inserted in [2.2), and a discrete Fourier transform with $\vec{q}=\frac{2 \pi}{L} \vec{m}$ is used, where $-\frac{L}{2}<m_{x / y} \leqslant \frac{L}{2}$ (assuming periodic boundary conditions). To cope with the singularity of $S_{\vec{q}}$ at $q=0$ in the discrete Fourier transform (which in the continuum formulation is integrable), one assigns a suitable value $\left|\vec{m}_{0}\right| \in(0,1)$ to the zero-frequency mode [35]. The choice does not affect the asymptotic scaling behavior but may still introduce deviations from the desired form, especially for strong correlations. For all cases considered, we adjusted $\left|\vec{m}_{0}\right|$ until the empirical mean diagonal correlation function normalized by the variance $\sigma^{2}$ of the distribution of random Gaussian correlated variables,

$$
\bar{C}(r)=\frac{1}{2 L^{2} \sigma^{2}}\left[\sum_{\vec{x}, \pm}\left(\varphi_{\vec{x}}-\bar{\varphi}\right)\left(\varphi_{\vec{x}+\vec{r}_{ \pm}}-\bar{\varphi}\right)\right]_{\mathrm{av}},
$$

was found in close agreement with equation [2.3]. Here, $\vec{r}_{ \pm}=(r, \pm r) / \sqrt{2}$ and the square bracket $[\ldots]_{\mathrm{av}}$ denotes the quenched disorder average. The mean value $\bar{\varphi}$ of the distribution $P(\varphi)$ is zero due to symmetry. An example of the resulting $\varphi_{\vec{x}}$ distribution is shown in the left-hand panel of figure 1 and $\bar{C}(r)$ is compared to the target function $C(r)$ of equation 2.3) in the left-hand panel of figure 2 The curves slightly level off for large $r$ due to the periodic boundary conditions.

At first glance, one might assume that the variance is $\sigma^{2}=1$ since the Fourier transform of the random Gaussian variables is scaled with the structure factor according to equation (2.2) and $\sigma^{2}=C(0)=$ $\int_{-\infty}^{\infty} \mathrm{d} \vec{q} S_{\vec{q}}=1$. However, this is only valid for the continuous Fourier transform in infinite space, which was considered for the analytical derivation of equation (2.4). In the FFM, we use this as an approximation and work on a finite lattice, discretizing $S_{\vec{q}}$ in the interval $q_{x / y} \in(-\pi, \pi]$. The lattice spacing is fixed and causes aliasing effects, while in the infinite-size limit, the resolution of $S_{\vec{q}}$ approaches the analytic result. This enables us to estimate the infinite-size variance as $\sigma^{2}(a)=\int_{-\pi}^{\pi} \mathrm{d} \vec{q} S_{\vec{q}} \leqslant 1$, where unity is approached for $a \rightarrow 0$.

In the second step, the thus generated $\varphi_{\vec{x}}$ are mapped to correlated discrete binary values, $s_{\vec{x}} \in\{0,1\}$, with a mean density $p$ of non-defect sites. To this end, a threshold $\theta$ is introduced such that sites with 

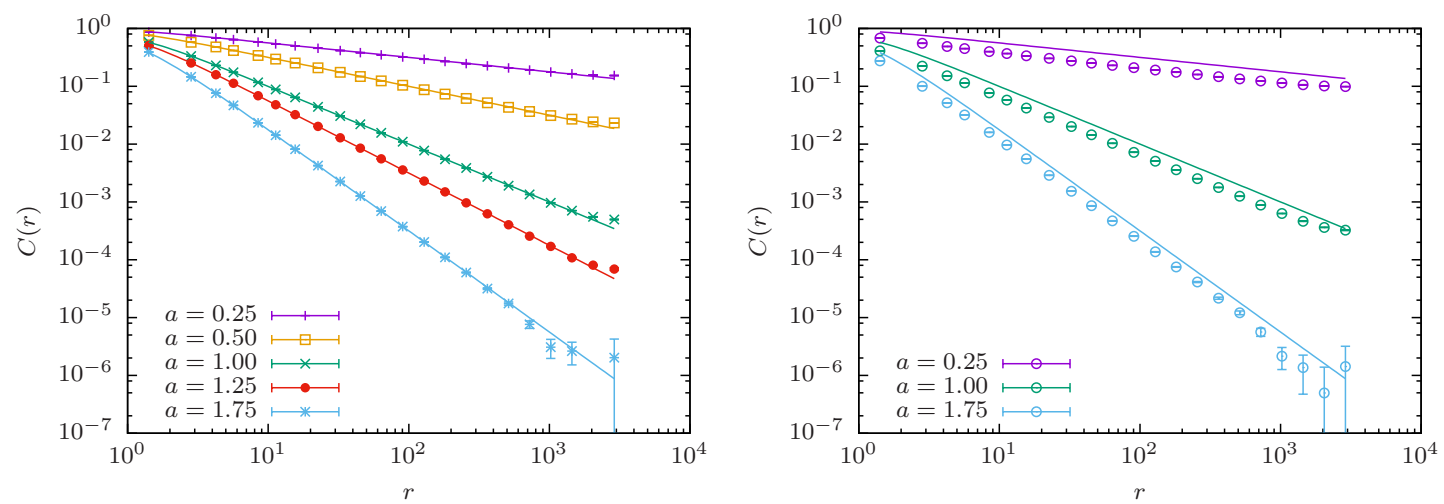

Figure 2. (Color online) Left-hand: measured diagonal correlation function $\bar{C}(r)$ (data points) compared to the input correlation function (2.3) (lines) of power-law correlated continuous Gaussian variables on a $2^{12} \times 2^{12}$ square lattice with periodic boundary conditions. Right-hand: the resulting diagonal correlation function of the mapped discrete disorder at the average percolation threshold $p=p_{\mathrm{c}}$ using equation 2.6. The lines show equation (2.3) normalized to the expected variance $p(1-p)$ to serve as a guide to the eye for the asymptotic behavior. All data are obtained as quenched average from $10^{5}$ replica.

$\varphi_{\vec{x}}>\theta$ are considered as defects. The threshold is tied to $p$ via

$$
p(\theta)=\int_{-\infty}^{\theta} P(\varphi) \mathrm{d} \varphi=\frac{1}{2} \operatorname{erfc}\left(\frac{-\theta}{\sqrt{2 \sigma^{2}(a)}}\right)
$$

where erfc is the complementary error function. Note that the fraction of defects on individual lattices significantly fluctuates for strong correlations and that here we consider the quenched disorder average $p_{L}=[p(\theta)]_{\text {av }}$ over all lattice realizations. The resulting pattern of the power-law correlated discrete binary variables $s_{\vec{x}}$ is shown in the right-hand panel of figure 1 As can be seen in the right-hand panel of figure 2 the correlations of the lattices mapped via equation (2.6) decay with the desired correlation exponent $a$ over a long range, though the amplitudes are somewhat diminished.

\subsection{Percolation threshold}

At low concentrations, clusters of the connected non-defect sites are of a typical size, despite the power-law correlations in the system. As for normal percolation, we can hence define a cluster correlation length $\xi$ that diverges as we approach the critical concentration $p_{\mathrm{c}}$. The universal exponent $v_{a}$ describing this divergence is supposed to be the same as for uncorrelated percolation, $v=4 / 3$ in two dimensions, as long as the correlations are sufficiently weak $(a \geqslant 2 / v)$ according to the extended Harris criterion [27], whereas for stronger correlations it is inversely proportional to $a$ [26, 27]:

$$
v_{a}=\left\{\begin{array}{ll}
v=4 / 3 & \text { for } a \geqslant 3 / 2 \\
2 / a & \text { for } \quad a<3 / 2
\end{array} \quad(d=2)\right.
$$

The value of $p_{\mathrm{c}}$ is not universal but depends on the lattice type and in general will also be affected by the presence of correlations (exceptions are bond percolation on the square lattice and site percolation on the triangular lattice, where $p_{\mathrm{c}}$ must remain $1 / 2$ for symmetry reasons [32]). Besides the asymptotic decay rate (i.e., the exponent $a$ ), short-range aspects of the correlation function and hence the method used to generate it should also play a role.

To estimate $p_{\mathrm{c}}(a)$, we analyzed at what concentration percolating clusters emerge for the first time. Following [47], we used the (horizontal) “wrapping criterion”, which has the benefit of relatively small finite-size effects. Accordingly, a cluster is defined as percolating if it closes back on itself across one specific boundary (e.g., horizontally). The threshold concentration was measured for a sample of $10^{5}$ disorder configurations for each lattice size $L=2^{i}, i=6, \ldots, 12$ for correlations between $a=0.125$ and $a=3$. 

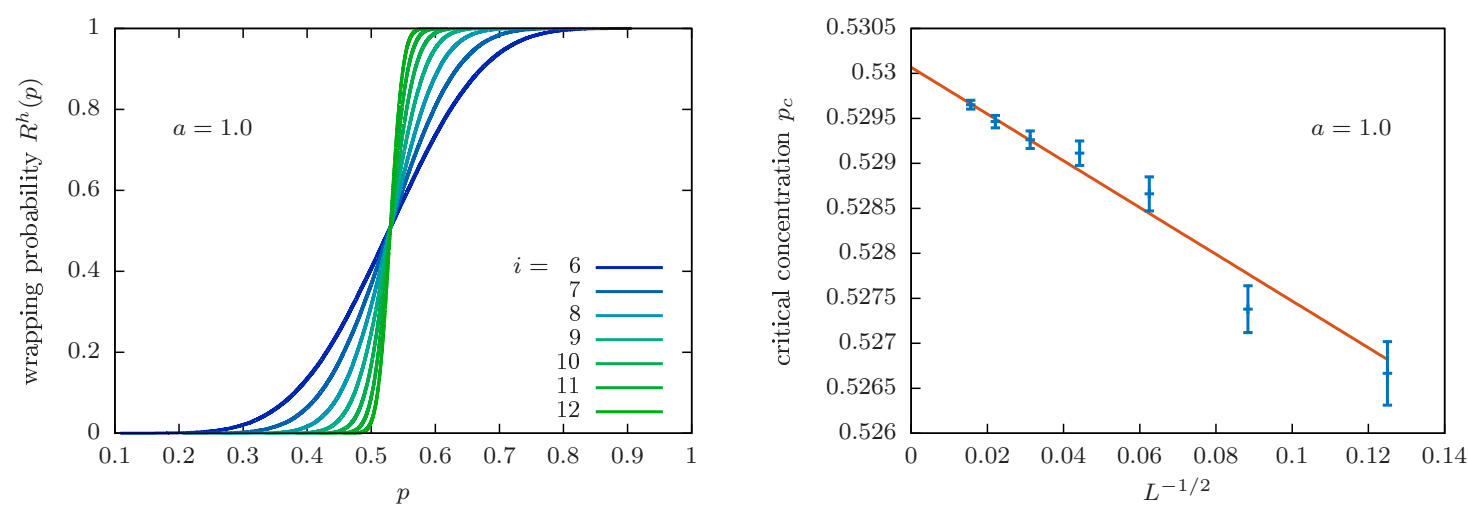

Figure 3. (Color online) Example for finite-size scaling of the percolation threshold for $a=1$ and lattice sizes $L=2^{i}, i=6, \ldots, 12$. Horizontal wrapping probabilities (left-hand) and critical concentrations (righthand) obtained from the quenched disorder average of the occupation numbers, $[p(\theta)]_{\mathrm{av}}$.

To do this, we adjusted the threshold $\theta$ following a simple bisection protocol until the sites with $\varphi \leqslant \theta$ just barely percolate. The result was then translated back to a concentration value using equation (2.6) and taking the quenched disorder average, $p_{L}=[p(\theta)]_{\mathrm{av}}$. We thus obtained the horizontal wrapping probabilities $R^{\mathrm{h}}(p)$ as the accumulated distributions shown in figure 3 (left-hand). With increasing size they become steeper, approaching a step function in the asymptotic limit. For stronger correlations, they are markedly more stretched because fluctuations in the system are more pronounced. The location of the intersection points on the $p$-axis appears to be a good estimator for $p_{\mathrm{c}}$ (cf. [47], figure 9).

To obtain a more controlled estimate, however, we used a standard finite-size scaling approach [14] for average threshold concentrations $p_{L, \mathrm{c}}$ :

$$
p_{L, \mathrm{c}}-p_{\mathrm{c}} \sim L^{-1 / v_{a}}
$$

where $v_{a}$ is given by equation 2.7). Plotting $p_{L, \mathrm{c}}$ vs. $L^{-1 / v_{a}}$, we hence obtain the percolation threshold $p_{\mathrm{c}}$ as the intersection of the best fit with the vertical axis as shown to the right in figure 3 for $a=1$ where $v_{a}=$ $2 / a=2$. The obtained results for $p_{\mathrm{c}}$ over a wide range of correlation strengths $a$ are plotted in figure 4 and listed below in table 1 . With increasing correlations (small $a$ ) the values for $p_{\mathrm{c}}$ tend towards $1 / 2$ as was observed in a previous study [31]. With decreasing correlations (large $a$ ), the value for uncorrelated disorder marked by the dashed line in figure 4 is approached surprisingly slowly, which shows how much the value of $p_{\mathrm{c}}$ is influenced by local (short range) properties of the system. As a check of our analysis

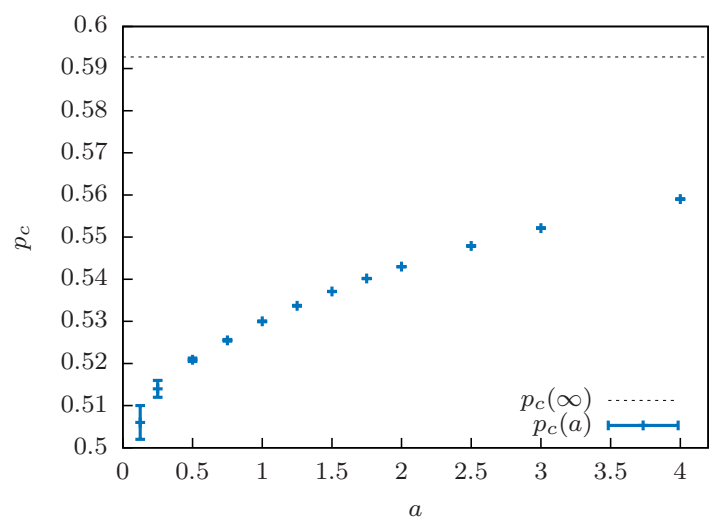

Figure 4. (Color online) Results of the percolation threshold $p_{\mathrm{c}}$ from the finite-size scaling extrapolation as a function of the correlation strength for the square lattice. The dashed line indicates the value for the uncorrelated case which is recovered for $a \rightarrow \infty$. 
method, we also looked at the completely uncorrelated case and found a good agreement with the known estimates [47. 48]. Interestingly, the values of the critical horizontal wrapping probabilities were all found very close to the (exactly known [49]) uncorrelated value $R_{\mathrm{c}}^{\mathrm{h}} \approx 0.52$.

\section{Random walk and diffusion exponents}

We now turn to the problem of diffusive random walks (RWs) in an environment with long-range correlated disorder. In the asymptotic limit, the mean square displacement as a function of the number of steps $N$ follows a power law:

$$
\left[\left\langle R^{2}\right\rangle\right]_{\mathrm{av}} \sim N^{2 v_{\mathrm{RW}}}
$$

with $v_{\mathrm{RW}}=1 / 2$ in any non-fractal medium. In fractal systems, however, random walks are usually subdiffusive with $v_{\mathrm{RW}}$ assuming a smaller, non-trivial value, and one defines the so-called walk (fractal) dimension as $d_{\mathrm{w}}=1 / v_{\mathrm{RW}}$. For two-dimensional uncorrelated critical percolation clusters, $d_{\mathrm{W}}$ has been measured very efficiently using the Lobb-Frank algorithm [50], yielding a very accurate estimate of $d_{\mathrm{w}}=2.8784$ (8) [51]. For any higher concentrations, the value of $d_{\mathrm{w}}$ is simply 2 since the system becomes homogeneous at large scales. In the presence of long-range correlated disorder, by contrast, the spatial distribution of defects is inhomogeneous on any length scale. This might affect the value of $d_{\mathrm{w}}$.

We used a simple exact enumeration method [52] to generate the probability densities of RWs. Specifically, we considered the "blind ant" rule where at each step, a diffusing particle (ant) randomly chooses its next position uniformly among all nearest neighbors. If it picks a defect, it will bounce back to its original position. The enumeration algorithm proceeds by successively calculating the probability distributions $P\left(\vec{x}_{i}, N\right)$ in order to find the walker at any site $\vec{x}_{i}$ after the $N$ th step from those after the $(N-1)$ th step:

$$
P\left(\vec{x}_{i}, N\right)=\frac{1}{4} \sum_{n} P\left(\vec{x}_{n}, N-1\right)+\frac{k}{4} P\left(\vec{x}_{i}, N-1\right),
$$

where the sum goes over all neighboring sites and $k$ is the number of adjacent defects. This algorithm has polynomial complexity $\propto N^{d_{l}+1}$, where $d_{l}$ ( $\approx 1.68$ for two-dimensional uncorrelated percolation) is the chemical dimension, so that very long walks can in principle be generated. From the knowledge of $P\left(\vec{x}_{i}, N\right)$, all moments or cumulants of the walk displacement, the return probability, etc. can be readily computed. However, to take a large disorder average (here, over $10^{5}$ replica) can still become very timeand memory-consuming, and we, therefore, limited our simulations to a maximum of $N=4096$ steps and focused in the analysis on the mean square displacement of the random walks.

We performed independent averages for walks of different maximal lengths $N_{i}$, which were chosen as rounded powers of $\sqrt{2}$. For each length, we only analyzed the final displacement, thus obtaining completely uncorrelated estimates for the average displacement after each length $N_{i}=2^{i / 2}, 0 \leqslant i \leqslant 24$. The results for varying correlation exponents $a$ and levels of concentration $p$ are shown in figure 5 For the uncorrelated case, shown on the top left, the behavior is as expected: for $p>p_{\mathrm{c}}$, the curves approach horizontal lines corresponding to normal diffusion, while the asymptotic behavior is characterized by a smaller exponent for $p=p_{\mathrm{c}}$. This also seems to be the case for $a=1.75$ (top right of figure 5), where we are still above the Harris boundary of $a=3 / 2$. For stronger correlations, it is hard to tell from these data whether or not the walks for $p>p_{\mathrm{c}}$ asymptotically follow the normal diffusive behavior. The curves for $a=1.25$ and $a=1.0$ seem to level off slightly, but the systems are too small to clearly reflect the asymptotic behavior. We did not find a convincing fitting approach for the data above $p_{\mathrm{c}}$, and the question whether long-range correlations can alter the walks' asymptotic scaling behavior even above criticality thus remains unsolved. The values at the critical concentration, on the other hand, are nicely described by power-law fits with confluent correction term:

$$
\left[\left\langle R^{2}\right\rangle\right]_{\mathrm{av}}=A N^{2 v_{\mathrm{RW}}}\left(1+b N^{-\Delta}\right) .
$$

Note that for uncorrelated percolation, the exponent of the correction term is known very accurately [53. 54), $\Delta=3 / 2 d_{\mathrm{w}}=0.5211(2)$.

The resulting estimates for the walk dimensions $d_{\mathrm{w}}=1 / v_{\mathrm{RW}}$ at criticality are plotted in figure 6 and listed in table 1. As can be seen, they are consistent with the estimate for uncorrelated percolation for 

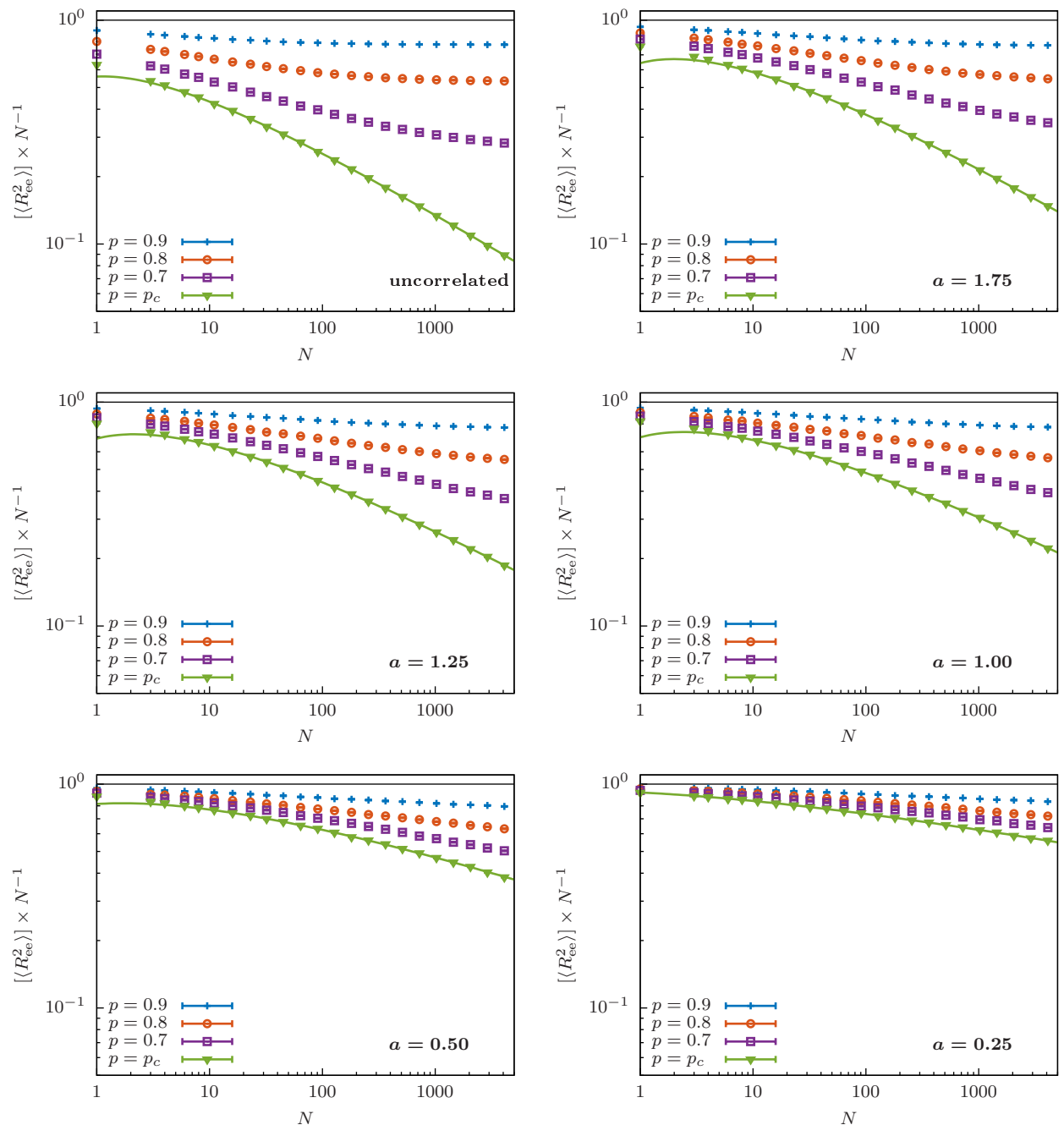

Figure 5. (Color online) Quenched disorder averages of the mean squared end-to-end distance per step for random walks on incipient percolation clusters for several degrees of correlation increasing from the top left to the bottom right panel and varying concentrations. The horizontal lines correspond to normal diffusion and the (green) lines for $p=p_{\mathrm{c}}$ are least-squares fits of equation (3.3).

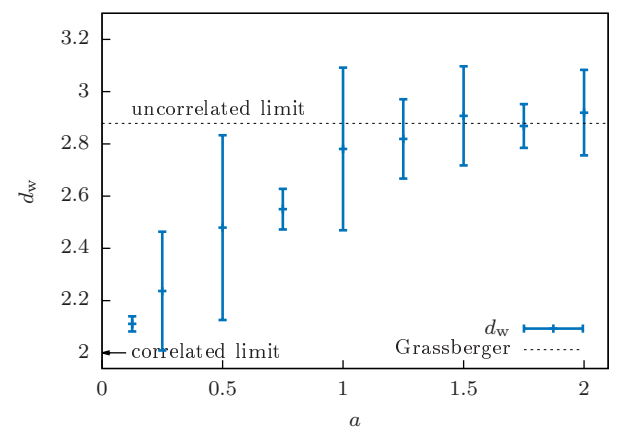

Figure 6. (Color online) Random walk dimension $d_{\mathrm{w}}$ at the percolation threshold $p_{\mathrm{c}}$ as a function of correlation exponent $a$. The horizontal dotted line shows the limiting value for the uncorrelated case when $a \rightarrow \infty$ [51]. 
Table 1. Results for the percolation threshold $p_{\mathrm{c}}$ and the walk dimension $d_{\mathrm{w}}$ for different correlation exponents $a$ at the percolation threshold $p_{\mathrm{c}}$. The errors provided refer to the fit errors.

\begin{tabular}{|l|l|l|}
\hline \hline$a$ & $p_{\mathrm{c}}$ & $d_{\mathrm{w}}$ \\
\hline \hline$\infty$ & 0.592746 [47,[48] & $\begin{array}{l}2.8784(8)[51] \\
2.92(5)\end{array}$ \\
\hline 3 & $0.55214(1)$ & $2.86(5)$ \\
\hline 2.5 & $0.54790(1)$ & $2.92(11)$ \\
\hline 2 & $0.54299(2)$ & $2.92(17)$ \\
\hline 1.75 & $0.54018(2)$ & $2.87(9)$ \\
\hline 1.5 & $0.53710(2)$ & $2.91(19)$ \\
\hline 1.25 & $0.5337(1)$ & $2.82(16)$ \\
\hline 1 & $0.5300(1)$ & $2.78(32)$ \\
\hline 0.75 & $0.5255(2)$ & $2.55(8)$ \\
\hline 0.5 & $0.5209(4)$ & $2.48(36)$ \\
\hline 0.25 & $0.514(2)$ & $2.24(23)$ \\
\hline 0.125 & $0.506(4)$ & $2.11(3)$ \\
\hline \hline
\end{tabular}

$a \geqslant 1.0$. For stronger correlations, the values decrease smoothly towards the full-lattice value of $d_{\mathrm{w}}=2$ for normal diffusion. This limit behavior is intuitively clear since any infinitely strongly correlated lattice will almost always either be fully occupied or empty. The agreement of $d_{\mathrm{w}}$ with the uncorrelated estimate for large $a$ is consistent with the theoretical predictions by Weinrib and Halperin [27] that above the extended Harris threshold $a=3 / 2$, the system effectively behaves as uncorrelated. However, the uncorrelated behavior of $d_{\mathrm{w}}$ persists even below the threshold down to $a \approx 1$. This is in agreement with observations for the fractal dimension, which also keeps its uncorrelated value well below the Harris threshold [32].

\section{Conclusions and prospects}

We investigated percolation on a square lattice with long-range correlated disorder. The correlations were generated using the improved Fourier filtering method (FFM) and carefully verified to decay according to the desired power law. The percolation threshold has been measured for different values of the correlation exponent $a$. The results strongly depend on the details of the method, cf. [31]. Here, we used the FFM as proposed by Makse et al. 35] combining an analytic continuum solution of the spectral density in infinite space with a discrete Fourier transform on a finite lattice. While this works well to the leading order, it introduces some subtle difficulties such as the adjustment of the zero mode or a variance of the desired distribution that deviates from unity. While this affects non-universal quantities such as the percolation threshold or the amplitudes in scaling laws, universal exponents such as the walk dimension $d_{\mathrm{w}}$ are not sensitive to such details.

We then performed an exact enumeration of random walks to investigate the properties of correlated percolation clusters as a medium for diffusion. The walk dimension $d_{\mathrm{w}}$ on critical clusters was determined as a function of the correlation exponent $a$. For weak correlations (large $a$ ), the uncorrelated behavior is recovered, and this behavior even seems to prevail below the threshold set by the extended Harris criterion (2.7). For increasing correlations (small $a$ ), $d_{\mathrm{w}}$ was found to approach the normal diffusion value of 2 . The results on super-critical clusters $\left(p>p_{\mathrm{c}}\right.$ ) are quantitatively less conclusive since the correlations strongly aggravate the finite-size effects. However, for the uncorrelated and the weakly correlated case, we qualitatively recovered the expected behavior of normal diffusion. In the strongly correlated case, persistence of anomalous diffusion cannot be ruled out from our data.

It would be very interesting to also study the behavior of self-avoiding walks on long-range correlated percolating clusters. For the uncorrelated case, there exist exact enumeration techniques that enable treatment of extremely long walks [55 [56]. While this approach becomes more demanding for an 
increasing correlation strength $(a \rightarrow 0)$, it should still be applicable to some extent. It could be supplemented by chain-growth Monte Carlo approaches such as PERM [57], which in the past were also used to study the uncorrelated system [20 21]. With some efforts, this would enable one to compare with field-theoretic predictions for polymers [15, 42,44].

\section{Acknowledgements}

The article is dedicated to Professor Yurko Holovatch on the occasion of his 60th birthday. The cooperation with the Lviv group was supported by an Institute Partnership Grant of the Alexander von Humboldt Foundation (AvH) under Grant No. 3.4-Fokoop-DEU/1117877, the Deutsch-Französische Hochschule (DFH) through the International Doctoral College “ $\llbracket$ " " Leipzig-Lorraine-Lviv-Coventry under Grant No. CDFA-02-07, and by the EU Marie Curie IRSES Network DIONICOS under Contract No. PIRSES-GA2013-612 707.

The work was funded by the Deutsche Forschungsgemeinschaft (DFG) via FOR 877 (project P9) under Grant No. JA 483/29-1 and Sonderforschungsbereich/Transregio SFB/TRR 102 (project B04). We are grateful for further support from the Leipzig Graduate School "BuildMoNa"-Building with Molecules and Nano-objects. JZ received financial support from the German Ministry for Education and Research $(\mathrm{BMBF})$ via the Bernstein Center for Computational Neuroscience (BCCN) Göttingen under Grant No. 01GQ1005B.

\section{References}

1. Pfeifer P., Avnir D., J. Chem. Phys., 1983, 79, 3558; doi 10.1063/1.446210

2. Avnir D., Farin D., Pfeifer P., Nature, 1984, 308, 261; doi 10.1038/308261a0

3. Bouchaud J.P., Georges A., Phys. Rep., 1990, 195, 127; doi 10.1016/0370-1573(90)90099-N

4. Malek K., Coppens M.O., Phys. Rev. Lett., 2001, 87, 125505; doi 10.1103/PhysRevLett.87.125505

5. Dullien F.A.L., Porous Media: Fluid Transport and Pore Structure, Academic Press, New York, 1979.

6. Sahimi M., Flow and Transport in Porous Media and Fractured Rock, VCH, Weinheim, 1995.

7. Skinner T.O.E., Schnyder S.K., Aarts D.G.A.L., Horbach J., Dullens R.P.A., Phys. Rev. Lett., 2013, 111, 128301; doi 10.1103/PhysRevLett.111.128301

8. Spanner M., Höfling F., Kapfer S.C., Mecke K.R., Schröder-Turk G.E., Franosch T., Phys. Rev. Lett., 2016, 116, 060601; doi 10.1103/PhysRevLett.116.060601

9. Bancaud A., Lavelle C., Huet S., Ellenberg J., Nucleic Acids Res., 2012, 40, 8783; doi 10.1093/nar/gks586

10. Höfling F., Franosch T., Rep. Prog. Phys., 2013, 76, 046602; doi 10.1088/0034-4885/76/4/046602

11. Harris A.B., J. Phys. C: Solid State Phys., 1974, 7, 1671; doi 10.1088/0022-3719/7/9/009

12. Harris A.B., Z. Phys. B: Condens. Matter, 1983, 49, 347; doi $10.1007 / B F 01301596$

13. Meir Y., Harris A.B., Phys. Rev. Lett., 1989, 63, 2819; doi 10.1103/PhysRevLett.63.2819

14. Stauffer D., Aharony A., Introduction to Percolation Theory, Taylor and Francis, London, 1992.

15. Blavatska V., von Ferber C., Holovatch Yu., In: Statistics of Linear Polymers in Disordered Media, Chakrabarti B.K. (Ed.), Elsevier, Amsterdam, 2005, 103-147.

16. Holovatch Yu., Condens. Matter Phys., 2006, 9, 237; doi 10.5488/CMP.9.2.237

17. Janke W., In: Order, Disorder and Criticality: Advanced Problems of Phase Transition Theory Vol. 3, Holovatch Yu. (Ed.), World Scientific, Singapore, 2012, 93-166.

18. Von Ferber C., Blavatska V., Folk R., Holovatch Yu., Phys. Rev. E, 2004, 70, 035104(R); doi $10.1103 /$ PhysRevE.70.035104

19. Janssen H.K., Stenull O., Phys. Rev. E, 2007, 75, 020801(R); doi 10.1103/PhysRevE.75.020801

20. Blavatska V., Janke W., EPL, 2008, 82, 66006; doi 10.1209/0295-5075/82/66006

21. Blavatska V., Janke W., Phys. Rev. Lett., 2008, 101, 125701; doi 10.1103/PhysRevLett.101.125701

22. Blavatska V., Janke W., J. Phys. A: Math. Theor., 2009, 42, 015001; doi 10.1088/1751-8113/42/1/015001.

23. Blavatska V., Janke W., Physics Procedia, 2010, 3, 1431; doi 10.1016/j.phpro.2010.01.202

24. Janssen H.K., Stenull O., Phys. Rev. E, 2012, 85, 051126; doi 10.1103/PhysRevE.85.051126

25. Blavatska V., Fricke N., Janke W., Condens. Matter Phys., 2014, 17, 33604; doi 10.5488/CMP.17.33604

26. Weinrib A., Halperin B.I., Phys. Rev. B, 1983, 27, 413; doi 10.1103/PhysRevB.27.413

27. Weinrib A., Phys. Rev. B, 1984, 29, 387; doi 10.1103/PhysRevB.29.387

28. Prudnikov V.V., Fedorenko A.A., J. Phys. A: Math. Gen., 1999, 32, L399; doi 10.1088/0305-4470/32/36/102 
29. Prudnikov V.V., Prudnikov P.V., Fedorenko A.A., J. Phys. A: Math. Gen., 1999, 32, 8587; doi $10.1088 / 0305-4470 / 32 / 49 / 302$.

30. Prudnikov V.V., Prudnikov P.V., Fedorenko A.A., Phys. Rev. B, 2000, 62, 8777; doi 10.1103/PhysRevB.62.8777

31. Prakash S., Havlin S., Schwartz M., Stanley H.E., Phys. Rev. A, 1992, 46, R1724(R); doi 10.1103/PhysRevA.46.R1724

32. Schrenk K.J., Posé N., Kranz J.J., van Kessenich L.V.M., Araújo N.A.M., Herrmann H.J., Phys. Rev. E, 2013, 88, 052102; doi 10.1103/PhysRevE.88.052102

33. Peng C.K., Havlin S., Schwartz M., Stanley H.E., Phys. Rev. A, 1991, 44, R2239(R); doi 10.1103/PhysRevA.44.R2239

34. Makse H.A., Havlin S., Stanley H.E., Schwartz M., Chaos, Solitons Fractals, 1995, 6, 295; doi 10.1016/0960-0779(95)80035-F

35. Makse H.A., Havlin S., Schwartz M., Stanley H.E., Phys. Rev. E, 1996, 53, 5445; doi 10.1103/PhysRevE.53.5445

36. Ballesteros H.G., Parisi G., Phys. Rev. B, 1999, 60, 12912; doi 10.1103/PhysRevB.60.12912

37. Blavatska V., von Ferber C., Holovatch Yu., Phys. Rev. B, 2003, 67, 094404; doi 10.1103/PhysRevB.67.094404

38. Blavatska V., Dudka M., Folk R., Holovatch Yu., Phys. Rev. B, 2005, 72, 064417; doi 10.1103/PhysRevB.72.064417

39. Blavatska V., Dudka M., Folk R., Holovatch Yu., J. Mol. Liq., 2006, 127, 60; doi 10.1016/j.molliq.2006.03.014

40. Ivaneyko D., Berche B., Holovatch Yu., Ilnytskyi J., Physica A, 2008, 387, 4497; doi 10.1016/j.physa.2008.03.034

41. Dudka M., Fedorenko A.A., Blavatska V., Holovatch Yu., Phys. Rev. B, 2016, 93, 224422; doi 10.1103/PhysRevB.93.224422

42. Blavatska V., von Ferber C., Holovatch Yu., Phys. Rev. E, 2001, 64, 041102; doi 10.1103/PhysRevE.64.041102

43. Blavatska V., von Ferber C., Holovatch Yu., J. Mol. Liq., 2001, 92, 77; doi 10.1016/S0167-7322(01)00179-9

44. Blavatska V., von Ferber C., Holovatch Yu., J. Phys.: Condens. Matter, 2002, 14, 9465; doi $10.1088 / 0953-8984 / 14 / 41 / 302$.

45. Bewerunge J., Ladadwa I., Platten F., Zunke C., Heuer A., Egelhaaf S.U., Phys. Chem. Chem. Phys., 2016, 18, 18887; doi $10.1039 /$ C6CP02559E

46. Press W.H., Teukolsky S.A., Vetterling W.T., Flannery B.P., Numerical Recipes: The Art of Scientific Computing, 3rd Edn., Cambridge University Press, Cambridge, 2007.

47. Newman M.E.J., Ziff R.M., Phys. Rev. E, 2001, 64, 016706; doi 10.1103/PhysRevE.64.016706

48. Ziff R.M., Phys. Rev. Lett., 1992, 69, 2670; doi 10.1103/PhysRevLett.69.2670

49. Pinson H.T., J. Stat. Phys., 1994, 75, 1167; doi 10.1007/BF02186762

50. Frank D.J., Lobb C.J., Phys. Rev. B, 1988, 37, 302; doi 10.1103/PhysRevB.37.302

51. Grassberger P., Physica A, 1999, 262, 251; doi 10.1016/S0378-4371(98)00435-X

52. Majid I., Ben-Avraham D., Havlin S., Stanley H.E., Phys. Rev. B, 1984, 30, 1626(R); doi 10.1103/PhysRevB.30.1626

53. Kammerer A., Höfling F., Franosch T., EPL, 2008, 84, 66002; doi 10.1209/0295-5075/84/66002

54. Ziff R.M., Phys. Rev. E, 2011, 83, 020107(R); doi 10.1103/PhysRevE.83.020107

55. Fricke N., Janke W., EPL, 2012, 99, 56005; doi 10.1209/0295-5075/99/56005

56. Fricke N., Janke W., Phys. Rev. Lett., 2014, 113, 255701; doi 10.1103/PhysRevLett.113.255701

57. Grassberger P., Phys. Rev. E, 1997, 56, 3682; doi 10.1103/PhysRevE.56.3682 


\section{Закони скейлінгу для випадкових блукань у далекосяжно-скорельованих невпорядкованих середовищах}

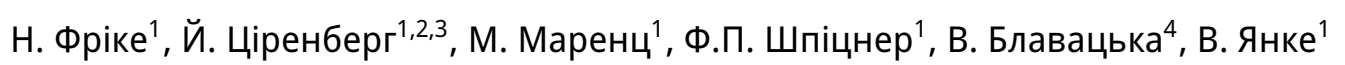

${ }^{1}$ Інститут теоретичної фізики, Університет Ляйпціґа, D-04009 Ляйпціґ, Німеччина

2 Центр обчислювальної нейробіології ім. Бернштейна, D-37077 Ґеттінґен, Німеччина

3 Інститут динаміки і самоорганізації Макса Планка, D-37077 Ґеттінґен, Німеччина

4 Інститут фізики конденсованих систем НАН України, вул. Свєнціцького, 1, 79011 Львів, Україна

Ми досліджуємо закони скейлінгу для дифузії у двовимірному середовищі із далекосяжно-скорельованим безладом шляхом точного підрахунку випадкових блукань. Невпорядковане середовище моделюється як перколяційний кластер із кореляціями, що спадають з відстанню згідно степеневого закону $r^{-a}$, згенерований за допомогою покращеного методу фільтрування Фур'є. Щоб охарактеризувати такий тип безладу, визначаємо поріг перколяції $p_{\mathrm{c}}$ шляхом дослідження імовірностей появи безмежного кластера. При $p_{\mathrm{c}}$ ми оцінюємо вимірність (суб-дифузивного) блукання $d_{\mathrm{w}}$ при різних значеннях кореляційного показни-

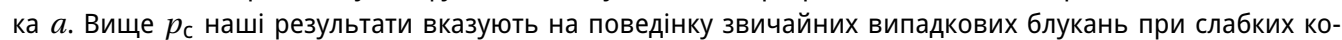
реляціях, в той час як не можна виключити аномальну дифузію у випадку сильних кореляцій, тобто при малих $a$.

Ключові слова: далекосяжно-скорельований безлад, критичні перколяційні кластери, випадкові блукання, точний підрахунок, закони скейлінгу 


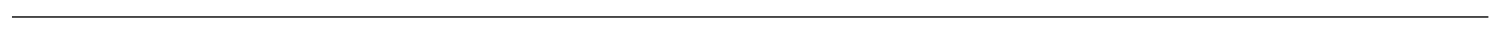

